\title{
Letters to the editor
}

Please submit letters for the editor's consideration within three weeks of receipt of Clinical Medicine. Letters should ideally be limited to 350 words, and sent by email to: clinicalmedicine@rcplondon.ac.uk

\section{Cardiac imaging to investigate suspected cardiac pain in the post-treadmill era}

Editor - I was pleased to see a review of cardiac imaging in patients with suspected cardiac chest pain, since this is a common problem with frequently an inconsistent approach to investigation (Clin Med 2014;14:475-81). However, I was saddened by its lack of proper perspective. X-ray computed tomography (CT) and cardiovascular magnetic resonance (CMR) were appropriately described, less so stress echocardiography, and myocardial perfusion scintigraphy (MPS) was relegated to three small paragraphs and was not mentioned as an option in the conclusion. In fact, MPS is the most commonly performed non-invasive test of coronary function in Europe, including the UK, and elsewhere. A brief letter is not the place to redress the balance but it may partly suffice to make the following points.

The diagnostic accuracy of each of the techniques is similar and individual comparative studies can give misleading results that may not be applicable outside the research setting. ${ }^{1}$ This equivalence has been recognised by the National Institute for Health and Care Excellence (NICE) and European Society of Cardiology (ESC) in their chest pain guidance. ${ }^{2,3}$ In truth, the diagnostic accuracy of a technique depends more upon the operator than the technique, which is why those who focus too closely within cardiac imaging may not see the full picture.

The prognostic accuracy of MPS in stable coronary disease has much greater evidence to support it than the other techniques, although the main determinant of events is the ischaemic burden rather than the technique used to assess it. The advantage of MPS is its ability to measure the ischaemic burden and that it can be used to predict which patients will do better with revascularisation than with medical therapy. Because of this, MPS is the only technique that has been shown to influence patient outcome for the better, albeit mainly in non-randomised trials. ${ }^{4-6}$

Although there are concerns related to ionising radiation, no technique is free of debateable harmful effects. ${ }^{7,8}$ MPS and CT benefit from methods of reducing radiation exposure to the equivalent of one year of background radiation, which may be below the threshold for significant harm, depending upon one's take on extrapolating the harm of higher exposures to levels below $10 \mathrm{mSv} .{ }^{9}$ For MPS, these techniques include the increased sensitivity of solid state gamma cameras and the short half-life radionuclides of positron emission tomography.

MPS has good evidence for its cost-effectiveness ${ }^{10,11}$ and the only prospective randomised trial comparing the techniques in the setting of stable chest pain showed that, in contrast to MPS, stress echocardiography and perfusion CMR are unlikely to be cost effective compared with invasive coronary angiography. ${ }^{12}$ Indeed, there was evidence of harm in the group randomised to CMR.

Mark Twain is dubiously credited with saying that, to the man with a hammer, every problem looks like a nail. Such an attitude in cardiac imaging may benefit the carpenter but it may be of less benefit for our patients.

S RICHARD UNDERWOOD

Professor of cardiac imaging, Imperial College London London, UK, and honorary consultant, Royal Brompton and Harefield NHS Foundation Trust, London, UK

\section{Pericardial mass and cardiac tamponade associated with Mycoplasma pneumoniae}

Editor - This interesting case report (Clin Med 2014;14: 549-50) raises the interesting issue of positive Mycoplasma serology and the interpretation of serology in general. The titre of the initial antibody, IgM, is the only Mycoplasma serology reported with the laboratory results in Table 1 . This is usually reported as a titre (eg 1 in 40) although here is expressed as units. Low titres of Mycoplasma IgM are often false-positive results, thought to be due to problems of cross-reactivity with polyclonal IgM. Even in scientific studies, specificity and sensitivity of the test is suboptimal, and depends on the assay used. ${ }^{1}$ IgG is more reliable but the reliability of this test is based on an increase in titre between the acute and convalescent phase. A single positive IgG alone simply means that the patient had been exposed to Mycoplasma infection at some point, possibly years earlier. To determine if Mycoplasma infection may have contributed to causation in this case, it is essential that the authors report the initial and follow-up titres of IgG. It is not clear from the text that the case was discussed with their local infection specialist. Had they done so, I am sure this specialist would have pointed out to them the complexities of interpreting Mycoplasma IgM and the difficulties in presuming that Mycoplasma infection was a contributing factor in the clinical illness reported here. It is critical that your readers are not misinformed on this clinical condition and the difficulties in interpreting positive serology.

ANNA GOODMAN

Locum consultant in infection, Guys and St Thomas' Hospital NHS Trust, London, UK 